\title{
e-Migrinter
}

$7 \mid 2011$

Réflexions croisées sur les migrations en Afrique de l'Ouest

\section{Les Maliens en France et aux États-Unis}

Trajectoires et pratiques transnationales dans des espaces migratoires différenciés

\section{Claire Boulanger et Kévin Mary}

\section{(2) OpenEdition}

\section{Journals}

Édition électronique

URL : https://journals.openedition.org/e-migrinter/869

DOI : 10.4000/e-migrinter.869

ISSN : 1961-9685

\section{Éditeur}

UMR 7301 - Migrinter

Édition imprimée

Date de publication : 1 septembre 2011

Pagination : 17-28

ISSN : 1961-9685

Référence électronique

Claire Boulanger et Kévin Mary, «Les Maliens en France et aux États-Unis », e-Migrinter [En ligne], 7 |

2011, mis en ligne le 07 septembre 2017, consulté le 20 mai 2021. URL : http://

journals.openedition.org/e-migrinter/869; DOI : https://doi.org/10.4000/e-migrinter.869 
 \\ Les Maliens en France et aux États-Unis : trajectoires et pratiques transnationales dans des espaces migratoires différenciés
}

\author{
Claire Boulanger \& Kévin Mary
}



epuis une vingtaine d'années, un nombre croissant d'auteurs

s'intéressent à la notion de transnationalisme en tant que paradigme qui permet de sortir de l'analyse unidirectionnelle du fait migratoire (qui oppose notamment pays d'émigration et pays d'immigration) et permet de comprendre l'existence de modes de vies qui transcendent les frontières (Marques, Góis, 2008).

Dans cette perspective, les migrants développent des formes actives de participation à des processus économiques, politiques, ou culturels, touchant à la fois la région ou le pays de départ et la région ou le pays de destination, réunis pour l'analyse en un espace social transnational

(Faist, 2000). C'est ce que nous entendons ici par « pratiques transnationales ». L'objectif de cet article est d'analyser ces pratiques à travers l'exemple des Maliens en France et aux États-Unis.
Pour des raisons historiques, les espaces migratoires reliant la France et les États-Unis au Mali sont différents : structurés avec la France; en construction avec les États-Unis. Si les divergences semblent à première vue "sauter aux yeux » dans cette comparaison entreprise de manière à mieux interroger deux terrains spécifiques, nous avons néanmoins pris garde de moduler systématiquement les échelles de comparaison de manière à mettre également en lumière les similitudes entre ces deux situations.

En effet, l'objectif est ici de dégager les invariants des pratiques transnationales d'une population émigrée, d'interroger l'unité et la densité des espaces sociaux où ces pratiques ont lieu, tout en restant attentif aux particularités de chacune des sociétés d'accueil, et aux origines sociales des migrants eux-mêmes. L'analyse fait ainsi ressortir des pratiques transnationales à la fois individuelles et collectives.

Après avoir brossé un court portrait de l'émigration malienne, il s'agira de cerner les influences des modèles sociétaux français et étasuniens sur les évolutions récentes de la présence malienne en leur sein, ainsi que sur les modes d'organisation de ces migrants. Enfin, l'analyse portera sur la comparaison des différentes pratiques transnationales (financières, matérielles, idéelles, etc.) des Maliens de France et des États-Unis. 


\section{Trajectoires migratoires : entre maintien des relations historiques et diversification des horizons}

\section{L'émigration malienne}

Les autorités maliennes parlent d'une population émigrée de près de 4 millions de personnes, soit près d'un tiers de la population malienne. Ce chiffre inclut non seulement les nationaux expatriés, mais aussi leurs descendants, qu'ils soient ou non de nationalité malienne. Néanmoins, les ambassades et consulats maliens, utilisant le seul critère de nationalité, estiment le nombre de leurs ressortissants présents à l'étranger à environ 920 000. De son côté, la Banque Mondiale qui mène des recherches principalement dans le but de canaliser les remises des migrants dans le système bancaire, estime en 2010 à un peu plus d'un million le nombre de Maliens émigrés ${ }^{1}$, sachant que le mode de calcul utilisé sousestime probablement le nombre de personnes ayant émigré dans les pays du Sud.

Or, à l'image des migrants subsahariens dans leur ensemble, les Maliens migrent peu en dehors de l'Afrique. Ainsi, le premier pays d'émigration des Maliens est la Côte d'Ivoire. Viennent ensuite le Burkina Faso, le Sénégal, le Gabon, etc. Ce tour d'horizon statistique fait donc ressortir un premier point commun de cette comparaison : les Maliens en France et aux États-Unis représentent une très faible part de l'émigration malienne; partir en Occident, émigrer, requiert d'importantes ressources, à la fois sociales et financières.

En France, le Ministère des Affaires étrangères estime la communauté malienne en 2009 à environ 80000 personnes, dont 48000 avec un titre de séjour ${ }^{2}$. Considérant

1 Fiche pays Mali disponible sur: http://siteresources.worldbank.org/INTPROSPECT S/Resources/334934-1199807908806/Mali.pdf

2 Source: http://www.diplomatie.gouv.fr/fr/payszones-geo $833 / \mathrm{mali} 350 /$ presentation-du- les flux, c'est-à-dire l'arrivée de migrants maliens sur le territoire français, on observe une croissance irrégulière mais continue des effectifs qui passent d'environ 400 personnes par an au milieu des années 1990 à environ 2000 au début des années 2000 pour enfin atteindre 4500 titres de séjour en 2009 (Prioux, 2010, p. 426).

Aux États-Unis, ces flux connaissent une croissance encore bien plus rapide : ils ont été multipliés par six entre 2000 et 2009, passant de 100 à près de 600 entrées annuelles ${ }^{3}$. Quant aux données sur les stocks de migrants, le Bureau du recensement américain estimait le nombre total d'immigrés maliens vivants sur le sol américain à un peu moins de 3000 personnes en 2000. Les Maliens des États-Unis sont donc beaucoup moins nombreux que ceux de France, ce qui explique en partie les contrastes observés ci-après.

\section{Des espaces migratoires différenciés}

\section{L'espace migratoire Mali - France : un couple migratoire fruit d'une longue histoire}

Après des décennies de colonisation, et donc de migration française au Mali, les migrations maliennes vers la France commencèrent au début du vingtième siècle, mais ne s'amplifièrent que dans les années 1960. En effet, la Guerre d'Algérie suscite une certaine méfiance vis-à-vis des travailleurs algériens et pousse les industriels à se tourner vers la main-d'œuvre venue d'Afrique noire. Au Mali, la multiplication des sècheresses fait peu à peu de la migration internationale un moyen de subsistance pour les communautés touchées. Les filières migratoires qui s'établissent et se structurent forment un couple migratoire entre les deux territoires. Les réseaux relient

\footnotetext{
mali 996/index.html

${ }^{3}$ Source : Yearbooks of immigration Statistics (1998-2009), Department of Homeland Security (DHS), Évolution du nombre annuel de migrants maliens admis aux ÉtatsUnis (1986-2009).
} 
deux régions en particulier : celle de Kayes, au sud ouest du Mali (qui fut la première région du Mali contemporain à être colonisée), et la région parisienne.

D'avantage fixée en France par des politiques migratoires restrictives et par le regroupement familial, la migration malienne vers l'Hexagone change de visage dans les années 1980. Nombre des hommes qui vivaient seuls dans les foyers sont rejoint par leurs familles et investissent de nouveaux espaces résidentiels. Ainsi, la structure même de ces filières est en évolution constante. Aujourd'hui, on observe la coexistence des cadres migratoires et post-migratoires avec les enfants de ces travailleurs qui n'ont pas eux-mêmes migrés ou ceux qui, arrivés à l'âge de la retraite, retournent au Mali.

En parallèle, venus effectuer leurs études universitaires en France, les intellectuels ouest-africains présents en France jusqu'à la fin des 1970, étaient pour beaucoup actifs dans la lutte pour les indépendances. Ils effectuaient un séjour de quelques années pour ensuite rentrer au Mali mener une carrière politique ou occuper des postes de cadres dans la fonction publique. Depuis les années 1980, la «défonctionnarisation » de la population active malienne, causée par les Plans d'Ajustements Structurels, pousse les intellectuels africains à rechercher d'autres d'opportunités professionnelles en restant dans le pays où ils ont fait leurs études. Une recherche portant sur le niveau de formation des immigrés maliens en situation régulière dans l'Union européenne des quinze en 1990, estime à seulement 5\% les personnes ayant un diplôme d'études secondaires (Docquier et Marfouk, 2005).

\section{L'espace migratoire Mali - États-Unis : une immigration récente en forte croissance}

La migration entre le Mali et les ÉtatsUnis remonte aux années 1960, elle concernait alors des étudiants. Il ne s'agissait alors que de quelques dizaines de personnes ${ }^{4}$. C'est au cours de cette période et surtout à partir de 1990 qu'elle se diversifie et se développe, notamment grâce à l'inauguration du programme de visa d'immigrant « diversité » qui favorise les pays à faible taux d'immigration vers les ÉtatsUnis, dont le Mali. Ce programme est en fait une loterie qui permet d'obtenir une "green card» et de gagner son statut de " migrant ». C'est donc depuis environ une vingtaine d'années que l'émigration malienne vers les États-Unis prend son essor.

Cette croissance peut être en partie éclairée par l'évolution des politiques migratoires françaises qui tendent à faire du contexte américain un cadre plus favorable à l'arrivée de migrants maliens. S'ils soulignent le fait que l'obtention d'un visa reposait presque uniquement sur les capacités financières des demandeurs, les interlocuteurs rencontrés aux États-Unis ${ }^{5}$ ont pour la plupart indiqué que l'obtention d'un visa ou d'une carte de séjour est aujourd'hui plus facile aux États-Unis qu'en France. Les lois qui conditionnent l'obtention de la nationalité française sont également plus restrictives que les lois américaines. Aux États-Unis, le droit du sol est appliqué strictement ${ }^{6}$ : toute personne née aux États-Unis, de parents étrangers ou

\footnotetext{
4 Source : Rapport Open Doors (1948-2009), Institute for International Education (IIE) : Évolution du nombre d'étudiants maliens aux États-Unis (1960-2009).

${ }^{5}$ Entre les mois de mars et juillet 2010, une vingtaine d'entretiens ont été menés à Washington DC et New York. L'enquête s'est concentrée sur la population étudiante, les leaders associatifs, les membres du corps diplomatique et les hauts cadres maliens travaillant à la Banque Mondiale.

${ }^{6}$ Bien qu'il soit régulièrement remis en cause, en particulier dans les États frontaliers du Mexique (Arizona, Texas, etc.).
} 
non, en est citoyen (selon le $14^{\text {ème }}$ amendement de la constitution des ÉtatsUnis).

\section{Sociétés d'accueil, projets migratoires, et conséquences sur les modes d'organisation communautaire}

\section{Le « rêve américain » et ses ambiguïtés}

Les États-Unis se sont construits selon le modèle d'une société où l'ascension sociale est possible pour tous, indifféremment de l'origine. Bien que ce mythe ait été invalidé depuis, la croyance dans le «rêve américain» est toujours tenace. Ce mythe trouvait déjà un écho fort, en Afrique, notamment, grâce à l'influence de la culture populaire afro-américaine. Il a potentiellement été amplifié récemment grâce à l'élection de Barack Obama.

Dans son dernier roman, Mammadou Mahmoud N'dongo décrit la façon dont l'un de ses personnages d'origine africaine en France voit changer l'image que l'on se fait de lui en arrivant aux États-Unis : «En France j'avais des origines africaines, c'est à New York que je suis devenu Noir» (2010, p.169). Les Africains arrivant aux États-Unis sont Noirs, au même titre que les Afro-américains auxquels ils sont assimilés. Ils deviennent en quelque sorte une "minorité invisible». Si cette appartenance à la communauté noire peut être utilisée pour certaines revendications (Salzbrunn, 2007), l'image idyllique des immigrés africains assimilés aux Afro-Américains doit être nuancée. Effectivement, la littérature scientifique souligne des rapports ambigus entre ces deux communautés (Mwakikagile, 2009). Ce constat est corroboré par des entretiens où les interlocuteurs font une distinction entre les catégories populaires et celles plus éduquées: "Avec les Afro-américains, il n'y a pas vraiment de rapports. On s'en méfie beaucoup en fait car ils prennent de la drogue, ce n'est pas notre milieu, ça ne nous arrange pas du tout, peu d'américains noirs sont instruits ou intellectuels.
Donc des fois cela arrive qu'on nous accuse dans la rue de les avoir vendus, par ignorance des choses ${ }^{7} »$.

\section{La France donne et renvoie une image dépréciée}

De son côté, la France n'est plus réellement considérée comme un pays «d'accueil». La persistance (voir le renforcement) des rapports de domination et de la discrimination met à mal le modèle méritocratique. La mobilité sociale reste difficile pour un Malien dans la société française, quelle que soit son origine sociale ou sa formation. Les «travailleurs immigrés » doivent prouver leur capacité à s'intégrer dans la République, comme le démontre la mise en place du "Contrat d'accueil et d'intégration » rendu obligatoire en 2006 ou encore les débats récents sur «l'identité nationale ».

Renvoyant une image dépréciative des migrants maliens, la France entraîne la dépréciation de sa propre image à leurs yeux, mais sans que cela ne remette en cause la pérennité des flux, protégés par des filières suffisamment denses.

\section{Vers un rééquilibrage des profils socio-économiques}

Les figures de l'accueil et de l'immigré propres à ces deux sociétés, associées aux coûts de la migration, ont crée deux " profils type » de migrants maliens : le travailleur peu qualifié en France, et le migrant hautement qualifié aux États-Unis.

Nous avons vu qu'en 1990, seul 5\% des Maliens d'Europe avaient un diplôme d'études secondaires. En 2000, cette même étude estime leur part à $16 \%$, ce qui marque une importante évolution des profils socioéconomique des migrants en question. Sur le continent américain, c'est la tendance inverse

\footnotetext{
7 Source: entretien réalisé à Bamako en mai 2009 auprès d'un migrant de retour des États-Unis.

8 Sur cette question, voir les travaux de Gérard Noiriel ou de Pap Ndiaye.
} 
qui se profile. On observe une sensible diminution des Maliens ayant un diplôme secondaire, au profit des moins scolarisés qui voient leur proportion passer de $7,6 \%$ à presque $20 \%$ en 10 ans (Docquier et Marfouk, 2005, tableau A.2-1 et A.2-2 ).

Les deux dernières décennies tendent donc vers un rééquilibrage des écarts de qualifications entre Maliens de France et Maliens des États-Unis. Cela est du, en France, au développement récent des politiques dites d' "immigration choisie », à l'image de la carte "Compétences et talents », un permis de travail destiné aux étrangers hautement qualifiés, à l'image du visa «H1-B» délivré aux États-Unis. Concernant la progression de l'immigration malienne moins qualifiée aux États-Unis, l'hypothèse la plus probable est celle du développement de réseaux de migrations de travail, particulièrement dans la ville de New York.

\section{Des projets migratoires contrastés}

Lacroix et al. (2008, p. 27) à propos des Sénégalais de France expliquent que "l'acte migratoire est inséré dans un dispositif social et familial qui contraint les acteurs à conserver des liens avec la communauté d'origine. Ce dispositif prémunit contre l'affaiblissement des liens sociaux entre les migrants et les personnes qui n'ont pas pu émigrer.» En effet, l'objectif premier de ces migrations est d'obtenir un emploi qui permettra d'envoyer de l'argent à sa famille, restée au village. Pour garantir la continuation du projet migratoire collectif, les aînés doivent s'assurer que les jeunes dépensent une part minimum de leurs revenus dans le pays de résidence. Bien entendu, ce contrôle social n'est pas sans faille. Peu à peu, en particulier avec le regroupement familial, certains individus échappent à la pression sociale du groupe, au profit de leurs parents les plus proches quoique cet éloignement n'aboutisse à la rupture totale qu'exceptionnellement. $A$ contrario, aux États-Unis, l'entrée par les élites a exclusivement mis en lumière des projets migratoires individuels.

$\mathrm{Si}$ les projets migratoires qui conduisent aux États-Unis ou en France sont relativement contrastés, il n'en reste pas moins que l'enjeu de la migration est comparable : il s'agit d'assurer l'ascension ou le maintien de son statut social au Mali, et ce, par la mobilité géographique.

\section{En France, une profusion d'associations de ressortissants maliens}

La vie associative malienne semble plus stable comparativement à celle d'autres communautés immigrées en France. Non pas en termes organisationnels (la durée de vie des associations n'étant pas forcement longue), mais par sa densité et sa continuité. En effet, elle n'est pas animée directement par des crises politiques et sociales maliennes, mais cherche à répondre à des besoins eux-mêmes constants, que ce soit en France ou au Mali. Christophe Daum (1998), estime que dans les années 1990, près de $70 \%$ des ressortissants maliens étaient membres actifs d'une association. Aujourd'hui néanmoins, de plus en plus de leaders de ces associations se plaignent que "les jeunes ne veulent plus payer», qu'il s'agisse de jeunes migrants ou de descendants de migrants nés en France.

Le fait est que ceux-ci ont souvent investi d'autres pans de la vie associative. Organisations officialisées auprès des autorités françaises, elles forment des nouveaux partenariats (ONG françaises, collectivités territoriales, institutions diverses). La mobilisation associative devient un moyen de faire valoir leur position au sein de la société française, que ce soit au travers d'actions ciblant leur communauté en France (soutien juridique/administratif, scolaire, etc.) ou tournées vers le Mali. Concernant les élites, nos entretiens font état de leur relatif non engagement dans les associations "communautaires", ce qui marquerait une évolution par rapport aux 
résultats de Abdoulaye Gueye (2001, p. 96) qui, dans les années 1980 et 1990, observait des intellectuels alors partie prenante de ces associations, souvent dans le but d'orienter leur activités. Par contre, nous avons pu observer que certains participent activement à la vie d'autres types d'associations, touchant des domaines culturels, professionnels ou se faisant le relais de partis politiques.

\section{Les développements récents des organisations communautaires maliennes aux États-Unis}

La communauté malienne de plus en plus nombreuse aux États-Unis s'organise et marque sa présence sur le territoire américain. L'inauguration du Centre culturel malien, en mars 2010, en est l'un des symboles forts. Cette ONG s'est donné, entre autres missions, celle de dispenser des cours d'histoire du Mali, de danses traditionnelles maliennes, ainsi que de langue bambara et de français. Situé à New York dans le quartier du Bronx, il s'agit du premier centre culturel malien à l'extérieur $\mathrm{du}$ pays. L'inauguration, quelques jours après, du siège de l'association des Maliens de New York (AMANY) est un autre symbole de la structuration de la présence malienne sur le sol américain.

Contrairement à la France et son modèle républicain de laïcité, l'approche communautaire de l'immigration aux ÉtatsUnis permet aux Maliens d'affirmer leur appartenance à la communauté musulmane des États-Unis. Aussi, dans le quartier de Harlem, les Ouest-Africains ont une mosquée à leur disposition.

Illustration $\mathrm{n}^{\circ} 1$ : Prière du vendredi aux abords d'une mosquée située à Little Sénégal (Harlem, New York).

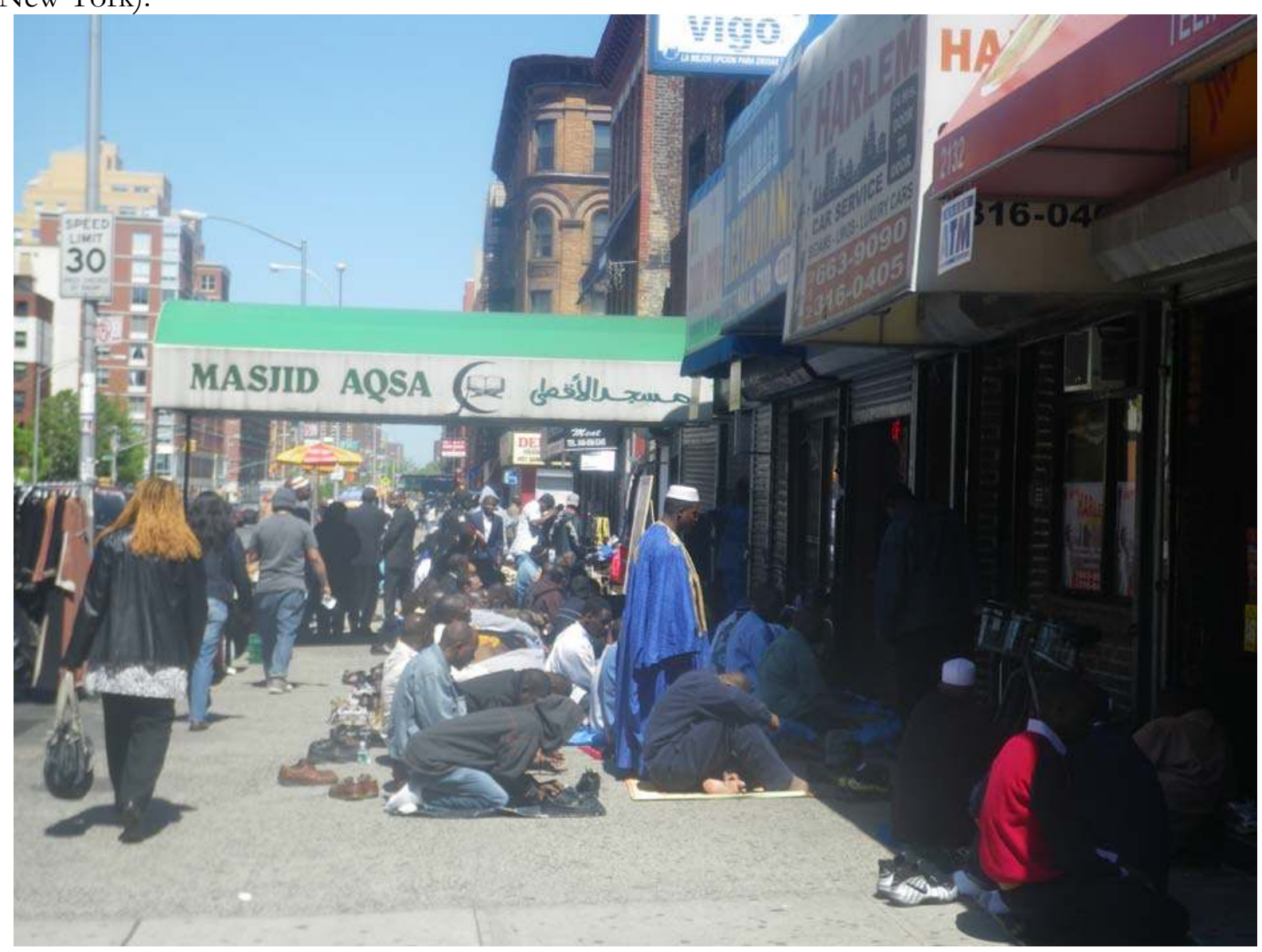

Source : Kévin Mary, Mai 2010. 
A propos des Sénégalais de Harlem, Monika Salzbrunn (2007) estime que l'identification de ces derniers à la communauté musulmane a été un élément ayant permis leur intégration dans la société américaine. Les autorités de ce pays n'hésitant pas à contacter les leaders religieux des différentes communautés pour leurs projets sociaux, d'éducation, mais aussi commerciaux. Il en résulte, selon cette même étude, un fort sentiment d'identification aux États-Unis, plus grand $\mathrm{du}$ moins que celui qu'ont les migrants sénégalais de Paris envers la France.

\section{L'État malien de plus en plus présent}

Après la chute du régime militaire de Moussa Traoré en 1991, l'État malien a modifié son «idéal national» pour faire des Maliens de l'extérieur une partie prenante de la nation, rompant avec le climat de suspicion qui régnait durant les années de dictature. Conscient des enjeux économiques et politiques que représentent les expatriés, l'État malien institutionnalise également la question migratoire en créant en premier lieu le Haut Conseil des Maliens de l'Extérieur (HCME), suivi de la création du Ministère des Maliens de l'Extérieur et de l'Intégration Africaine (MMEIA), crée " afin de promowvoir et protéger les intérêts des Maliens établis à l'étranger $\gg$.

Mais c'est surtout par leur présence auprès des migrants que les représentants du pouvoir politique malien accroissent leur influence sur la diaspora. Cette présence s'illustre à la fois dans les pays d'accueil lors de visites à Paris ou à New York, ou au travers des partis politiques et de leur représentation en France, particulièrement actives en ce moment à l'approche de l'élection présidentielle prévue en 2012. Cette présence auprès des migrants se fait aussi au Mali avec par exemple les discours d'inauguration de structures créées par des migrants, manière de démontrer la reconnaissance de l'État malien, de façon à la fois symbolique et concrète. Un autre exemple est l'organisation du «Forum de la diaspora » (dont la première édition c'est tenue en 2003) permettant de réunir à Bamako les représentants des différentes communautés de Maliens vivant à l'étranger, dans le but affiché d'écouter leur doléances.

Portant le nom de «Centre Culturel Malien Madame ATT Lobbo Traoré », en hommage à la première dame du Mali qui en a financé une partie, ce lieu est dénoncé par certains membres de la communauté malienne des États-Unis comme étant trop personnifié et donc difficile à s'approprier par l'ensemble de cette communauté. Ce sont logiquement des proches de la Première dame du Mali qui dirigent le centre.

Cette politisation accrue des Maliens de l'extérieur se reflète également par la présence de plus en plus importante du gouvernement malien dans les organisations dites « non gouvernementales».

Le Haut Conseil des Maliens de l'extérieur (HCME) a initialement été pensé comme une structure fédératrice des associations et amicales des Maliens de l'extérieur. Mais il est aujourd'hui contrôlé de facto par le Ministère des Maliens de l'extérieur, seul habilité à reconnaître les compétences des Hauts Conseils dans chaque pays d'immigration.

Ces dynamiques d'institutionnalisation et de politisation peuvent être pensées à la fois comme une consolidation du pouvoir étatique malien au-delà de ses frontières, mais aussi et conséquemment, comme un étouffement et une récupération des initiatives émanant de la société civile.

\footnotetext{
9 Source : http://www.maliensexterieur.gouv. $\mathrm{ml} /$ contenu page.aspx?pa $=24$
} 


\section{Origines sociales et pratiques transnationales}

Avant d'aborder les différents types d'échanges qui contribuent à densifier les espaces sociaux transnationaux qui nous occupent, rappelons que d'un côté ou de l'autre de l'Atlantique, les migrants maliens sont membres de familles transnationales ou dispersées (Razy, 2007) et restent donc en communication quasi permanente avec le Mali par le biais du téléphone et d'Internet.

\section{Flux financiers}

La Banque Africaine de Développement estime à 456 millions d'euros le montant global des transferts à destination du Mali pour l'année 2005. L'intérêt de cette étude, contrairement à celles de la Banque Mondiale, est qu'elle prend en compte les flux monétaires informels, dont elle évalue la part à $73 \%$. Cette étude a également montré qu'avec près de 300 millions d'euros par an, la France est de loin le premier pays émetteur de ces transferts. Néanmoins, le rapport souligne l'existence d'autres "corridors» et cite la Côte d'Ivoire avec 84 millions d'euros envoyés au Mali, l'Espagne avec 47 millions d'euros, et enfin les États-Unis avec 20 millions d'euros.

Chez certaines catégories d'interlocuteurs rencontrés, les transferts financiers se font à l'inverse, au départ du Mali. C'est en particulier le cas de ceux dont les parents financent entièrement les études, un cas de figure assez courant aux ÉtatsUnis. Rien n'était envoyé au Mali par les étudiants d'origine aisée rencontrés, si ce n'est des cadeaux de manière ponctuelle, lors de vacances scolaires passées au Mali. Pourtant, certains d'entre eux ont travaillé pendant leurs études mais l'argent qu'ils gagnaient était plutôt destiné à améliorer leurs conditions de vie sur place.

Notons enfin qu'en France tout au moins, les associations contribuent également à ce type d'échanges puisque les cotisations de leurs membres financent la construction d'infrastructures et d'équipements collectifs, voire les salaires des personnes qui y travaillent.

\section{Les échanges matériels}

Concernant les flux commerciaux, les échanges sont de volumes très variables, depuis le grand commerçant qui utilise le fret aérien et les conteneurs marchands, jusqu'au «commerce à la valise » pratiqué par des individus circulant entre le Mali et le pays tiers, le plus souvent, en parallèle d'une autre activité professionnelle.

Concernant les flux non commerciaux, nous pouvons premièrement noter le cas du transport simple. $\mathrm{Ni}$ commerçants, ni donateurs, nombre de migrants maliens, sont des porteurs d'« ordonnances », c'est-àdire de médicaments, le plus souvent accompagnés de leur prescription: une ordonnance malienne qui aura donc préalablement fait le «trajet» jusqu'au lieu de résidence du migrant. Cette pratique touche tous les profils, à l'exception de ceux aux origines sociales les plus aisées, qui pourront se permettre de s'approvisionner en médicaments directement depuis Bamako. Le caractère courant de cette pratique est lié à la fois aux coûts d'achats qui est le plus souvent assuré par les migrants, mais aussi à la qualité des produits, réputée meilleure dans les pays d'immigration. Pour transporter ces précieux colis, on choisira de préférence quelqu'un de son réseau social proche, mais en cas d'urgence, un inconnu, rencontré aux terminaux des avions à direction de Bamako, pourra devenir le précieux intermédiaire.

Une autre partie des échanges matériels est composé de dons à destination du Mali. La vie associative des Maliens de France étant plus développée que celle de leurs homologues américains, les pratiques collectives y sont plus courantes. Ainsi, les associations villageoises ou de quartier sont 
connues pour leurs collectes de dons (matériel scolaire, médical, agricole, informatique) qui seront le plus souvent envoyés par conteneurs vers la localité concernée. On peut souligner que cette pratique semble de moins en moins populaire chez les bénéficiaires, qui font souvent face à du matériel usagé, obsolète ou inadapté. De leur côté, certains membres de l'élite économique, mettent en place des œuvres caritatives, souvent en leur nom propre.

\section{Échanges techniques et symboliques}

Enfin, des échanges immatériels participent également à la densification de ces espaces sociaux étendus au-delà des frontières. Les transferts de compétence des émigrés maliens au profit de structures maliennes sont pour partie institutionnalisés. Depuis 1998, le programme TOKTEN (en français «Transfert des connaissances par l'intermédiaire des expatriés »), initialement sous l'égide du PNUD, soutient l'organisation et le financement de missions d'enseignement à l'Université de Bamako. Dans sa nouvelle version "élargie», le programme encadre des missions d'appui technique dans les secteurs de l'agriculture, de la santé et des PME/PMI, auprès des structures maliennes qui en font la demande. Le répertoire dans lequel les coordinateurs du programme trouvent les coordonnées des intellectuels expatriés s'est beaucoup élargi ces dernières années. De 290 personnes recensées en 2007 (dont 87 en France et 39 aux États-Unis), elles étaient 525 à travers le monde en 2010 (dont 115 en France, 52 aux États-Unis) ${ }^{10}$. Mais le programme a des moyens limités. Environ 500 missions (de 2 à 3 semaines environ) ont eu lieu jusqu'à présent, prenant la forme de cours magistraux, de conférences scientifiques, d'encadrement de thèses et de formation continue. En parallèle, des membres de

\footnotetext{
10 Source : Répertoire de la diaspora intellectuelle et scientifique du Mali disponible au 3 janvier 2011 sur http://www.maliens-exterieur.gouv.ml/ documentation/repertoire dispora mali.pdf
}

l'élite scientifique sont amenés, par exemple par l'intermédiaire de réseaux professionnels, à aller former des personnes au Mali, donnant également un contenu cognitif aux échanges.

L'influence des migrants peut aussi se faire sentir à l'échelle des localités (villages, quartiers ou communes). Par exemple, la création d'associations locales au Mali est souvent motivée par la nécessité de gérer sur place les projets organisés et financés par les migrants. Ces créations ne vont pas sans entrainer des tensions avec les structures existantes (chefferies, mairies, etc..) en raison de la concurrence pour des ressources, financières, matérielles ou humaines qui demeurent limitées. Dans une certaine mesure, la structuration de la société civile au Mali se fait donc pour répondre aux besoins organisationnels des migrants impliqués dans le développement de leur région d'origine.

La thèse de Stéphanie Lima (Lima, 2003) qui a suivi le processus de décentralisation dans la Région de Kayes, décrit le rôle important que les associations inter-villageoises de migrants ont pu jouer dans le découpage des communes, et donc dans la territorialisation de l'espace malien.

A l'échelle nationale, nous avons vu comment l'État malien a su se rapprocher de sa diaspora. On peut également relever que les migrants, de par leurs organisations ou leurs réseaux personnels, pourront influencer les politiques. En France, une illustration intéressante de ce phénomène est celle de la Mutuelle des Maliens de l'extérieur. Au tout début des années 1990, soucieux de l'accès aux soins de leurs proches, des ressortissants à Paris d'un village malien demandent conseil à la Fédération des Mutuelles de France sur la faisabilité d'une mutuelle dont ils seraient les adhérents, mais dont les ayants droits se trouveraient au Mali. L'étude de faisabilité, en partie financée par le Ministère de la Coopération, révèle le besoin, pour assurer 
la couverture des soins à distance, de s'appuyer sur une structure relais au Mali. De cette évaluation naîtra, en 1996, l'Union Technique de la Mutualité malienne, organisme d'appui à l'ensemble des mutuelles maliennes existantes ou en formation. Ainsi doté, le Mali est le pays de la région où cette forme d'économie solidaire est la plus développée, et ce en conséquence de l'impulsion donnée par des migrants.

\section{Conclusion}

De part leur histoire, le nombre de personnes concernées et leur structuration, les espaces migratoires entre le Mali et la France et entre le Mali et les États-Unis sont très contrastés. Si le recul n'est pas encore suffisant, en raison du développement récent des migrations maliennes vers les ÉtatsUnis, il semblerait néanmoins que la tendance soit à l'aplanissement de ces différences. D'abord parce que les migrants eux-mêmes diversifient leurs stratégies migratoires. Ensuite car les politiques migratoires (distinctes des politiques d'intégration) françaises et américaines tendent à se rapprocher.
Ce gommage progressif incite à réfléchir à la notion de diaspora appliquée au contexte malien. Peut-on parler de diaspora malienne aujourd'hui? La réponse semble différente selon les définitions qui peuvent être données de ce terme. Si l'on s'en tient à la définition relativement restrictive qu'en a donné Emmanuel Ma Mung (2000), il semblerait que non: les liens entre les différents pôles d'émigration semblent en effet encore trop peu importants. Aussi, pourrions-nous plutôt évoquer des «formes diasporiques en construction » au regard de la diversification croissante des pôles d'arrivée de l'émigration malienne.
Claire Boulanger

Doctorante

MIGRINTER/CEDEM, claire.boulanger@,voila.fr

Kévin Mary

Doctorant

MIGRINTER/ESO-CAEN, kevin.mary@unicaen.fr 


\section{Bibliographie}

Daum, Christophe (1998) Les associations de Maliens en France (migrations, développement et citoyenneté), Paris, Karthala, 253 p.

Devriendt, Arthur (2008) Les Maliens de Montrenil, des "Quêteurs de Passerelles ", Paris, Université Paris 1 Panthéon-Sorbonne. Mém. Master I : Géogr. : Paris : 2008.

Docquier, F.; Marfouk, A. (2005) International Migration by Educational Attainment (1990-2000), in Ozden C.; Schiff M., International migration, remittances, and the brain drain, Washington DC, World Bank, pp. 40-41.

Faist, Thomas (2000) The Volume and Dynamics of International Migration and Transnational Social Spaces, Oxford, Clarendon Press, 380 p.

Gueye, Abdoulaye (2001) Les intellectuels africains en France, Paris, L'Harmattan, 272 p. (Collection Sociétés Africaines et Diaspora).

Lacroix, Thomas; Sall, Leyla; Salzbrunn, Monika (2008) Marocains et Sénégalais de France: permanences et évolution des relations transnationales, Revue européenne des migrations internationales, vol. $24 \mathrm{n}^{\circ} 2$, pp. 2343.

Lima, Stéphanie (2003) Découpage entre espace et territoire: la fin des limites? La fabrique des territoires communaux dans la Région de Kayes, Mali, Poitiers, Université de Poitiers, 532 p. Th. Doct. : Géogr. : Poitiers : 2003.

Ma Mung, Emanuel (2000) La diaspora chinoise, géographie d'une migration, Paris, Orphys, 175 p. (GéOphrys).
Marques, José Carlos ; Góis, Pedro (2008) Pratiques transnationales des Capverdiens au Portugal et des Portugais en Suisse, Revue européenne des migrations internationales, vol. 24 $\mathrm{n}^{\circ} 2$, pp. $147-165$.

Mwakikagile, Godfrey (2009) Africans and African Americans: Complex Relations: Prospects and Challenges, Dar es Salaam, New Africa Press, 216 p.

N'Dongo, Mamadou Mahmoud (2010) La géométrie des variables, Paris, Gallimard, 301 p. (Continents noirs).

Prioux, France; Mazuy, Magali; Barbieri, Magali (2010) L'évolution démographique récente en France: les adultes vivent moins souvent en couple, Population, vol $65 \mathrm{n}^{\circ} 3$, pp 421-474.

Razy, Élodie (2007) En Afrique et hors de ses frontières: la famille dispersée soninké aujourd'hui, Representation of the African Family of the 21 st century, Leiden.

Salzbrunn, Monika (2007) Localising Transnationalism: Researching political and cultural events in a context of migration, Conference "Transnationalisation and Development(s): Towards a North-South Perspective", Center for Interdisciplinary Research, Bielefeld.

Sayad, Abdelmalek (1999) La double absence. Des illusions de l'émigré aux souffrances de l'immigré, Paris, Seuil, 437 p. (Liber).

Thierry, Xavier (2004) Évolution récente de l'immigration en France et éléments de comparaison avec le Royaume-Uni, Population, vol 59 n5, pp 725-764. 